\title{
Periorbital Necrotising Fasciitis
}

\author{
GEOFFREY E. ROSE, ${ }^{1}$ DAVID J. HOWARD,${ }^{2}$ MARK R. WATTS ${ }^{1}$ \\ London
}

\begin{abstract}
Summary
Three cases of periorbital necrotising fasciitis are described, one occurring in a three-year-old child. The cases in adults required debridement of necrotic tissue, in one of whom there was extensive disease involving the face and orbital fat.

It is probable that the early stages of this condition are under-recognised; the importance of early signs and intensive treatment of this life-threatening disease are illustrated.
\end{abstract}

Necrotising fasciitis is an ischaemic necrosis of subcutaneous tissues (fascia), generally due to an overwhelming infection with $\beta$-haemolytic Streptococcus pyogenes. ${ }^{1,2}$ The disease has a rapid onset, often within a few days of minor breaks in the skin, and spreads extensively and rapidly through subcutaneous fascial planes.

The condition occurs most frequently in the limbs or the abdominal wall, where it has been given various terms, such as haemolytic streptococcus gangrene, ${ }^{3}$ gangrenous or necrotising erysipelas, suppurative fasciitis and Fournier's gangrene. Periorbital necrotising fasciitis is reported rarely, with a total of only 16 cases cited in a recent review. ${ }^{1}$ The low incidence in the head and neck might be attributed to the good blood-supply to these tissues, but some early cases might go unrecognised because they resolve, albeit slowly, during treatment with high-dose broad-spectrum antibiotics.

Three cases are presented, which display a spectrum of the disease. In all cases a large number of $\beta$-haemolytic Streptococci were isolated from necrotic periorbital subcutaneous tissues and in two cases debridement of periorbital (and, in one case, facial and intraorbital) tissues was required.

\section{Case Reports}

Case 1

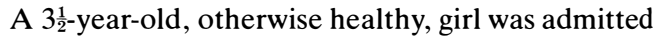
to the referring hospital with a 24-hour history of periorbital pain, general malaise, lethargy and a rapidly increasing severe periorbital swelling. For three days prior to admission, she had been treated with oral nystatin for perineal candidiasis and for two days had symptoms of an infection of the upper respiratory tract. Her right eyelids were closed by gross erythematous swelling, but her eye movements were normal and there was no relative afferent pupillary defect. A discharge from the right lids cultured large numbers of Group A $\beta$-haemolytic streptococci. She was treated with topical chloramphenicol and high-dose intravenous ampicillin and flucloxacillin.

During the first 12 hours of admission, the swelling of the right eyelid worsened, with development of an area of skin necrosis and purulent discharge from the subcutaneous tissues (Fig. 1); the left eyelids also became red and swollen. The intravenous therapy was continued at this hospital, together with local cleaning of the discharging sinus. There was a rapid improvement in her systemic state and

From ${ }^{1}$ Moorfields Eye Hospital, City Road, London EC1V 2PD: ${ }^{2}$ Royal National Hospital for Throat, Nose and Ear, Grays Inn Road, London WC1X 8DA.

Correspondence to: Mr. G. E. Rose, MS, FRCS, FCOphth, Moorfields Eye Hospital, City Road, London EC1V 2PD. 


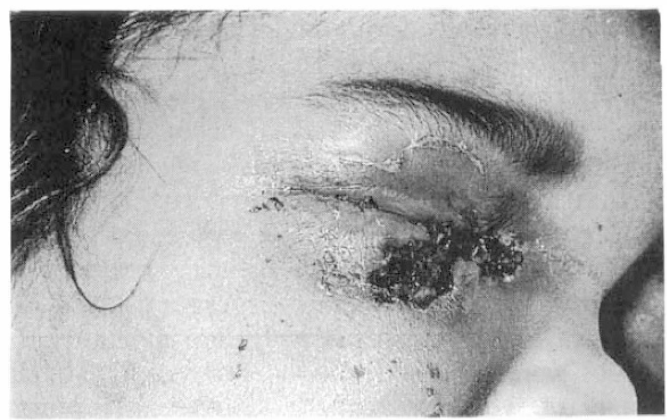

Fig. 1 (Case 1). Extensive Streptococcal preseptal cellulitis with necrosis of subcutaneous fat, discharging through a sinus of the right lower eyelid.

the preseptal infection and tissue necrosis resolved over three weeks.

Subsequent investigation did not reveal any abnormality of immunocompetence.

\section{Case 2}

A $2 \mathrm{~cm}$ brow laceration, requiring sutures, was sustained by a 50 -year-old man during a fall. The patient had a history of high alcohol intake and the accident occurred whilst he was drunk. Over the next three days his right eyelids became very painful and the eye closed due to extreme swelling. $\mathrm{He}$ presented to the referring hospital with signs of systemic infection, bilateral lid swelling and necrosis of the right upper eyelid (Fig. 2); there was marked cervical lymphadenopathy. Group A $\beta$-haemolytic Streptococci were cultured in large numbers from the discharge, together with some Staphylococcus aureus.

He was treated with high-dose intravenous benzyl penicillin, cefuroxime and metronidazole and the signs of his systemic illness improved. The brow wound was reopened and necrotic tissue debrided from the surrounding area to allow free drainage of the subcutaneous spaces. The necrotic skin was also debrided from the right upper eyelid and the tissues healed slowly, by secondary intention, over one month.

The patient, an itinerant labourer, was lost to follow-up.

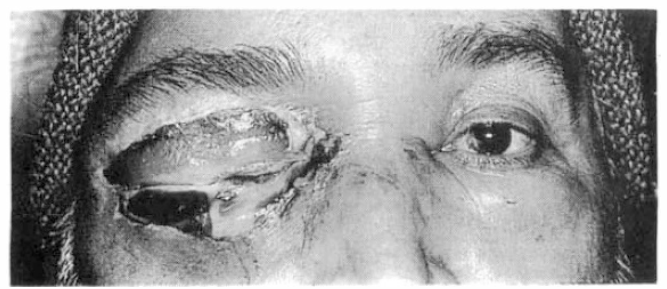

Fig. 3 (Case 3). Necrosis of the right eyelids 3 days after a minor eyelid laceration.

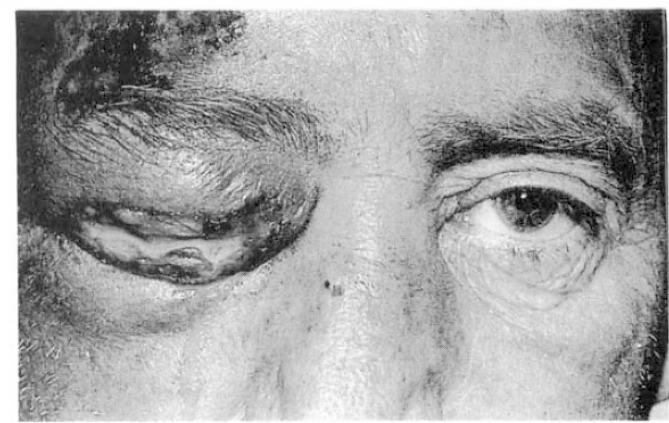

Fig. 2 (Case 2). Necrosis of the right upper eyelid skin and necrotising fasciitis of the scalp and face, 4 days after a brow laceration.

Case 3

Three days prior to admission, a 50-year-old man fell at home and sustained a $5 \mathrm{~mm}$ laceration of the right lower eyelid, which was cleaned and dressed at a local Casualty department. He was otherwise fit, but had a past history of high alcohol intake. Swelling of the eyelids and periorbital pain increased over two days and the skin of the right upper and lower eyelids sloughed during the 12 hours prior to admission.

On admission, the visual acuity of the affected side was $6 / 12$, with no relative afferent pupillary defect and a good range of ocular movements. Necrosis of the right eyelids was present, with sparing of the lid margins and lash lines (Fig. 3); oedema and cellulitis extended over the face and there was marked cervical lymphadenopathy. The patient was systematically unwell, with fever $\left(40^{\circ} \mathrm{C}\right)$, dehydration, proteinuria and macroscopic haemoglobinuria. Plain $x$-rays showed soft tissue swelling, but no gas or foreign bodies in the tissues.

Initial debridement was performed (Fig. 4) and Group A $\beta$-haemolytic Streptococci were isolated in very large numbers from the tissues and the pur-

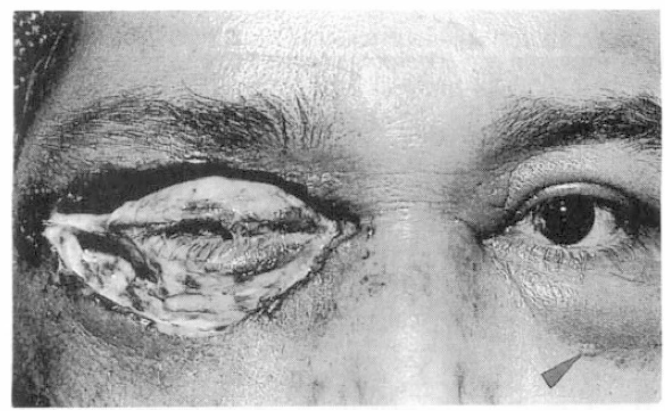

Fig. 4 (Case 3). Patientafterinitialtissue debridement without anaesthesia. There is vesicle formation in the oedematous skin of the left lower eyelid (arrow). 


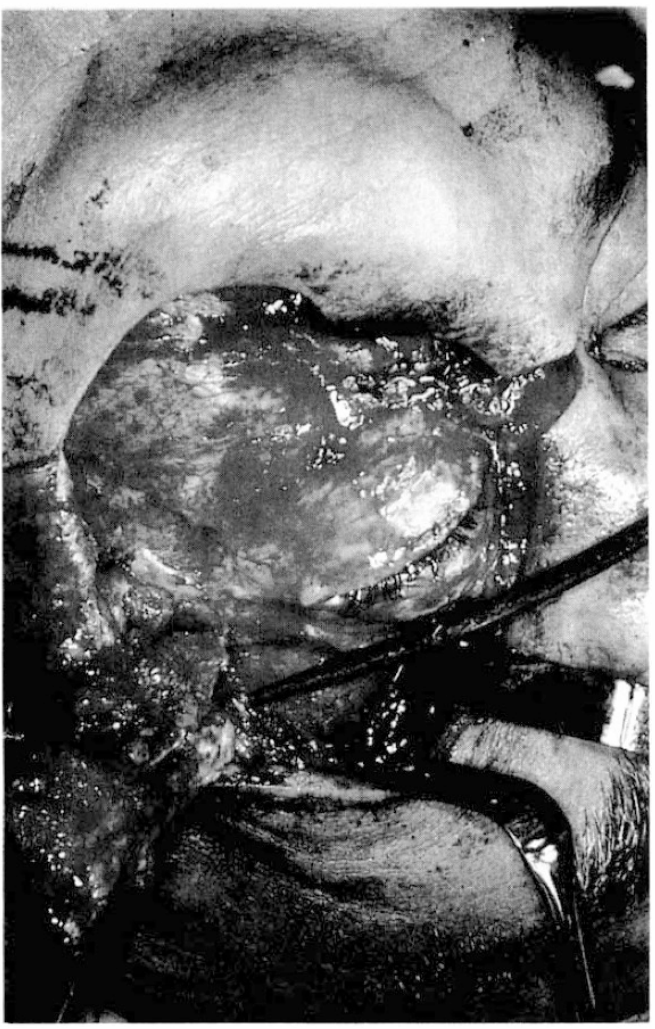

Fig. 5 (Case 3). Right side after debridement of periorbital tissues: Necrosis of the orbital septum and anterior orbital fat has occurred.

ulent discharge. Intravenous cefuroxime and metronidazole were started, at maximum dosage, and benzyl penicillin $(1.8 \mathrm{~g}$, four hourly) added when microbiology results were available. With rehydration and intravenous antibiotics, there was a considerable improvement in the general condition of the patient and the haemoglobinuria and

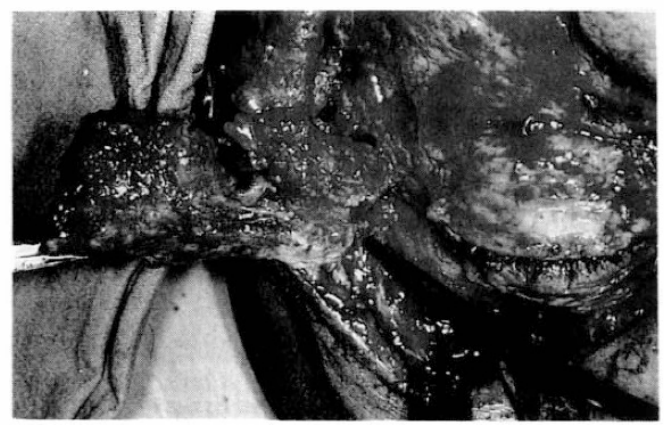

Fig. 6 (Case 3). Resection of non-perfused premaxillary and preauricular musculature. proteinuria resolved without development of acute renal failure.

Major exploration of the head and neck under general anaesthesia was delayed for 24 hours because of the severe septicaemia, dehydration and haemoglobinuria-with associated risk of renal failure. Exploration of the left periorbital tissues was considered mandatory because periorbital swelling had increased before surgery and a cutaneous vesicle had appeared (Fig. 4); the subcutaneous tissues were very oedematous, but viable. There was extensive necrosis of the right orbital septum and orbital fat, but sparing of the tarsal plates and lid margins (Fig. 5). Preauricular and premaxillary musculature was avascular and was described (Fig. 6) through the periorbital defect, a superior sublabial incision and a parotidectomy incision. Areas of debridement were cleaned and dressed with hydrogen peroxide packs. The deep and superficial cervical tissues and the carotid sheath were explored through the inferior transverse limb of the parotidectomy incision and the tissues found to be inflamed and oedematous, but without evidence of necrosis. Loose closure of the neck was performed,

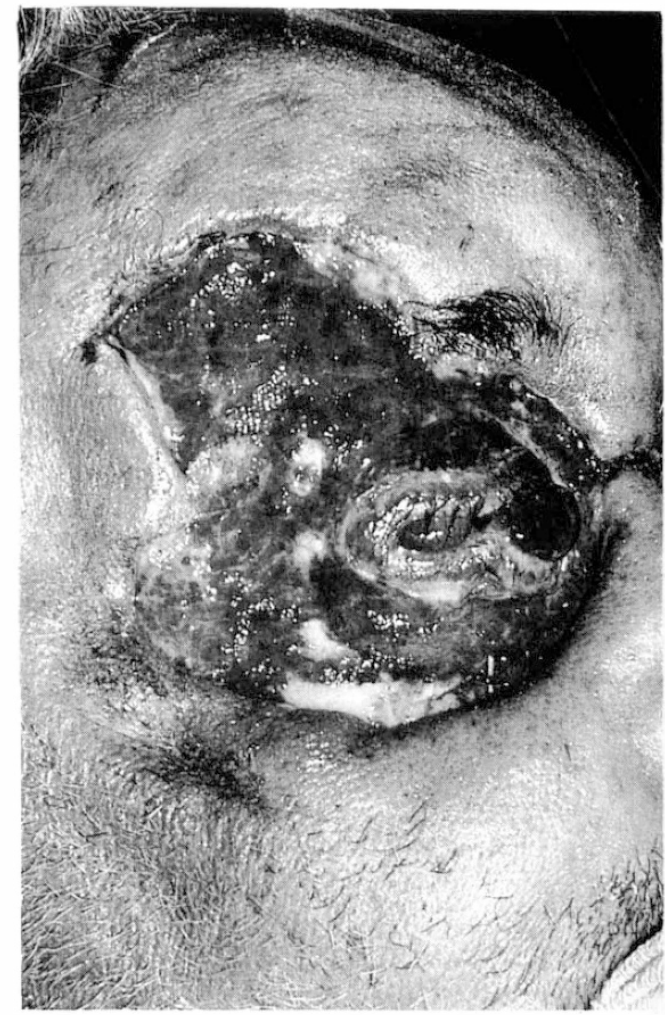

Fig. 7 (Case 3). Formation of granulation tissue at 2 weeks after major debridement of orbital and facial tissues. 


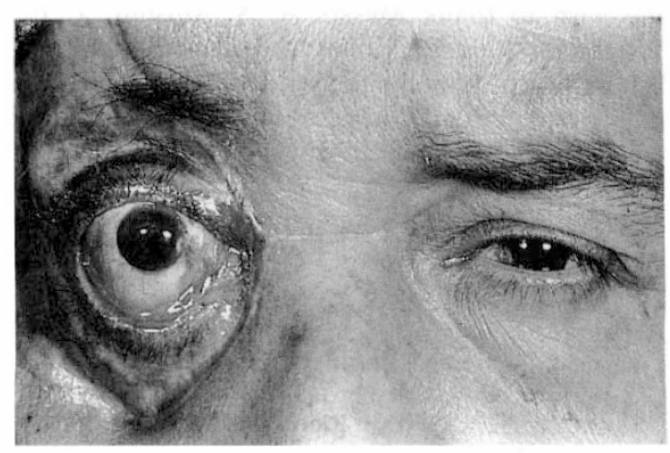

Fig. 8 (Case 3). Two months after debridement and later skin-grafting. Surgery to reduce the palpebral aperture is planned when tissue perfusion has further improved.

with insertion of broad corrugated drains, but the periorbital and sublabial incisions were left open.

The inflammation settled slowly on systemic therapy, which was continued (with reducing doses) over ten days, and the areas or orbital tissue loss granulated over two weeks (Fig. 7). Skin grafts were applied to the granulation tissue with the right upper tarsal plate placed on prolonged traction towards the cheek. Levator myotomy was not performed because this muscle was considered to provide the only significant vascular perfusion to anterior tissues.

Most full-thickness skin grafted successfully onto the tarsal plates and granulation tissue and all partial-thickness skin survived on the periosteum. Debridement of some non-viable graft was required and Group A Streptococci were isolated from this debris; the patient being off therapy at that time. Systemic phenoxymethylpenicillin was started and the affected areas healed by secondary intention (Fig. 8).

There are no corneal complications from the lagophthalmos, but surgery is planned to reduce the palpebral aperture when the tissues have had further time to establish adequate perfusion.

\section{Discussion}

The three cases presented show a spectrum of periorbital necrotising fasciitis, although the patients had several features in common.

In all cases there was extensive preseptal cellulitis, from which $\beta$-haemolytic Streptococcus pyogenes were cultured in very large numbers. There were clinical signs of systemic illness in all patients and, in contrast to previous reports, ${ }^{1,2}$ cervical lymphadenitis was prominent in the three cases. Intravascular haemolysis due to Streptococcal septicaemia caused macroscopic haemoglobinuria in Case 3 , but, with adequate hydration, did not result in acute renal failure. In all cases, the signs and symptoms of systemic infection improved within a day of starting high-dose intravenous antibiotics. In Case 3, cutaneous Group A Streptococci were isolated after the patient ceased antibiotic therapy: We now consider it, therefore, imperative to continue antibiotic therapy until all breaches of the skin are healed.

Necrosis of preseptal subcutaneous tissues occurred in all patients. The necrosis was limited in the mildest case (Case 1) to a discharge of pus and liquified fat from the right lower lid (Fig. 1) and the condition settled with antibiotic therapy alone. With more severe disease (Case 2), fat necrosis occurred beyond the area of skin necrosis in the right upper lid (Fig. 2); after local debridement and systemic antibiotics, the tissue defect slowly healed by secondary intention. In Case 3 , necrosis of the subcutaneous tissues and fascia extended up to $8 \mathrm{~cm}$ beyond the area of obvious skin necrosis and destroyed the frontal and premaxillary musculature (Figs 5 and 6). At debridement, there was complete ischaemia of the involved tissues with no bleeding from small vessels. Ischaemia both prevents tissue accumulation of bactericidal concentrations of antibiotics and provides a hypoxic environment for infection by anaerobic bacteria, as reported in other series. ${ }^{4,5}$ Necrosis of the orbicularis oculi muscles, orbital septum and anterior fat of the orbit occurred in Case 3 (Fig. 5) and loss of these structures is exceptional, ${ }^{1}$ a manifestation of the severity of disease in this case.

The degree of subcutaneous necrosis was, in all cases, of greater extent than suggested by changes in the overlying skin. The skin overlying areas of severe inflammation or subcutaneous necrosis may be hypoaesthetic, have poor vascular perfusion and a purple hue showing vesicle formation (Fig. 4). Such cutaneous changes warrant skin incision, along the lines of local vascular supply, and exploration of the subcutaneous tissues (Fig. 5). In Case 3, clinical signs in the face and neck were so extensive that exploration of the neck was performed.

In all cases necrosis occurred soon after 
relatively minor injury, either in a person unwell at the time (Case 1) or chronically debilitated by a past (Case 3 ) or present (Case 2) history of high alcohol intake. The lid laceration in Case 3 was small and the brow laceration in Case 2 had been previously cleaned and sutured; in neither case was a foreign body retained in the wound. The child (Case 1) might have innoculated her preseptal tissues, through a periorbital skin-scratch, during eye-rubbing.

These three cases illustrate the speed with which preseptal cellulitis, caused by $\beta$-haemolytic Streptococci entering through minor or unrecorded breaches of the thin lid skin, can progress to extensive necrosis of subcutaneous tissues. Whereas very high dose systemic antibiotics may lead to a slow resolution of very early cases of necrotising fasciitis, widespread and early debridement of dead tissue is imperative in many cases of this life-threatening condition.
We wish to thank Messrs A. S. Mushin, J. E. Wright and J. R. O. Collin for permission to publish details of patients (Cases 1, 2 and 3, respectively) under their care and also the staff of the Department of Medical Illustration, Moorfields Eye Hospital.

\section{References}

${ }^{1}$ Kronish JW and McLeish WM: Eyelid necrosis and periorbital necrotising fasciitis. Report of a case and review of the literature. Ophthalmology 1991, 98: 92-8.

${ }^{2}$ Walters R: A fatal case of necrotising fasciitis of the eyelid. Br J Ophthalmol 1988, 72: 428-31.

${ }^{3}$ Meleney FL: .A differential diagnosis between certain types of infectious gangrene of the skin: with particular reference to haemolytic streptococcus gangrene and bacterial synergistic gangrene. Surg Gynecol Obstet 1933; 56: 847-67.

${ }^{4}$ Rea WJ and Wyrick WJ Jr. Necrotizing fasciitis. Ann Surg 1970, 172: 957-64.

${ }^{5}$ Giuliano A, Lewis F Jr, Hadley K et al.: Bacteriology of necrotizing fasciitis. Am J Surg 1977, 134: 52-7. 